\title{
ON THE ROLE OF ELECTRON QUANTUM TUNNELING IN CHARGING OF DUST GRAINS IN COMPLEX PLASMA
}

\author{
YU.O. TYSHETSKIY, S.V. VLADIMIROV
}

PACS 52.27.Lw

(C) 2011

School of Physics, University of Sydney

(Sydney, NSW 2006, Australia; e-mail: y. tyshetskiy@ physics. usyd.edu. au)

\begin{abstract}
The role of the quantum tunneling effect in the electron accretion current onto a negatively charged grain immersed in isotropic plasma is analyzed, within the quasiclassic approximation, for different plasma electron distribution functions, plasma parameters, and grain sizes. It is shown that the contribution of the quantum tunneling into the grain charging is small (negligible) for relatively large (micron-sized) dust grains in plasmas with electron temperatures of the order of a few eV, but becomes important for nanosized dust grains (tens to hundreds $\mathrm{nm}$ in diameter) in cold and ultracold plasmas (electron temperatures $\sim$ tens to hundreds of Kelvin degrees), especially in plasmas with depleted high-energy "tails" in the electron energy distribution.
\end{abstract}

Complex plasmas - plasmas with dust particles (grains) in them [1-3] - owe their complexity, to a large extent, to the fact that the charging process of a dust grain embedded in a plasma is sensitive to the plasma parameters and to the proximity of other grains. Understanding the physics of grain charging is thus important for understanding complex plasmas.

The most commonly used model for finding the equilibrium charge of a grain immersed in plasma is the Orbital Motion Limited (OML) model [4], in which the electron and ion currents from plasma onto the grain are found by analyzing particle orbits and determining whether they intersect the grain, using classical mechanics. However, under certain conditions, quantum mechanical effects may become important, especially for electrons, and may lead to a significant change of these currents and, hence, of the equilibrium grain charge. Examples of additional currents induced by quantum effects include the electron photoemission current (if the grain is illuminated by sufficiently energetic photons) or thermionic and/or field electron emission currents from the grain to the surrounding plasma. These processes of spontaneous and field-assisted tunneling of thermal electrons out of negatively charged grains into plasma and the associated emission current densities have been well studied in the literature (see, e.g., [5] and references therein). However, the inverse process of quantum tunneling of plasma electrons onto a negatively charged grain, which might, under favorable conditions, significantly increase the rate of electron accretion from plasma onto the grain, has received no proper attention, with an exception of Ref. [6] where the cross-section for electron collisions with a spherical grain at low energies has been calculated quantummechanically.

The aim of this work is thus to calculate the additional current associated with the quantum tunneling of plasma electrons, that are classically forbidden to overcome the repulsive potential barrier, onto the negatively charged grain. We compare this additional quantum tunneling current with the classical electron current from plasma onto the grain and analyze how this additional current affects the self-consistent equilibrium grain charge for different plasma parameters and grain sizes.

We consider a spherical grain of radius $r_{0}$ immersed in an isotropic plasma with electrons and positive singly ionized ions. As the grain interacts with its environment, by absorbing electrons and ions from the plasma and emitting electrons via processes such as photo and/or thermionic emission, it acquires an equilibrium net charge. Here, we assume this charge to be negative, which is normally the case due to a higher mobility of plasma electrons compared to plasma ions, and a relatively minor contribution of electron emission processes from the grain to plasma.

To determine the electron accretion current onto the grain, we consider the radial motion of electrons in the stationary central field of a spherical negatively charged grain in isotropic plasma, governed by the effective potential $U_{\text {eff }}(r, l)=U(r)+\hbar^{2} l(l+1) / 2 m_{e} r^{2}$, where $l$ is the electron's quantum angular momentum number (angular momentum with respect to the center of the grain). In what follows, we consider the quasiclassical approximation of electron's motion in the grain's field (the criterion of validity of the quasiclassical approximation will be given below). An electron with total energy $E$ and the absolute value of angular momentum $\hbar \sqrt{l(l+1)}$, com- 
ing toward the grain from infinity, will hit the grain of radius $r_{0}$ if $E \geq U_{\text {eff }}\left(r_{0}, l\right)$. If $E<U_{\text {eff }}\left(r_{0}, l\right)$, it encounters a potential barrier of the width $r_{1}-r_{0}$, where $r_{1}$ is the classical turning point of the electron defined from $U_{\text {eff }}\left(r_{1}, l\right)=E$. In classical mechanics, the electron cannot penetrate this barrier, and is reflected back to infinity. But, in quantum mechanics, the electron can tunnel through the barrier onto the grain with some nonzero probability $w_{t}$. In the quasiclassical approximation, this probability can be obtained in the form [7]

$w_{t}(E, l)=\exp \left\{-\frac{2}{\hbar} \int_{r_{0}}^{r_{1}} \sqrt{2 m_{e}\left[U_{\mathrm{eff}}(r, l)-E\right]} d r\right\}$.

The corresponding "classical" electron current onto the grain, i.e., the current due to electrons with $E \geq$ $U_{\text {eff }}\left(r_{0}, l\right)$ that are classically allowed to hit the grain, is

$$
I_{\text {clas }}^{(e)}=\frac{4 \pi^{2} e}{m_{e}^{3}} \sum_{l=0}^{\infty} \hbar^{2}(2 l+1) \int_{U_{\text {eff }}\left(r_{0}, l\right)}^{\infty} d E f_{e}^{(-)}(E, l) w_{t}(E, l)
$$

with $U_{\text {eff }}\left(r_{0}, l\right)=U\left(r_{0}\right)+\hbar^{2} l(l+1) / 2 m_{e} r_{0}^{2}$, and $w_{t}(E, l)=1$ (as for electrons classically allowed to hit the grain $\left.w_{t}(E, l)=1\right)$, where $f_{e}^{(-)}(E, l)$ denotes the distribution of plasma electrons coming toward the grain, $e$ is the electron charge. The current on the grain due to electrons with $E<U_{\text {eff }}\left(r_{0}, l\right)$, which we here call the "tunneling" current, is then given by the same formula, but with the integral over $E<U_{\text {eff }}\left(r_{0}, l\right)$ :

$I_{\mathrm{tun}}^{(e)}=\frac{4 \pi^{2} e}{m_{e}^{3}} \sum_{l=0}^{\infty} \hbar^{2}\left(2 l+1 \int_{0}^{U_{\mathrm{eff}}\left(r_{0}, l\right)} d E f_{e}^{(-)}(E, l) w_{t}(E, l)\right.$

with the tunneling probability $w_{t}(E, l)$ defined by (1). Note that, in classical mechanics, the tunneling probability for electrons with $E<U_{\text {eff }}$ is zero [one can see this by formally taking the limit $\hbar \rightarrow 0$ in (1)], and thus $I_{\text {tun }}^{(e)}=0$, as expected.

The tunneling probability $w_{t}(E, l)$ can be evaluated analytically for the Coulomb (i.e., unscreened by plasma) potential of the grain, $U(r)=\alpha / r$, with $\alpha=$ $Z_{d} e^{2}$, where $Z_{d}$ is the charge of the grain in units of electron charge. Introducing the Coulomb units of mass, length, time, and energy as $m_{e}, \hbar^{2} /\left(\alpha m_{e}\right), \hbar^{3} /\left(\alpha^{2} m_{e}\right)$, and $\alpha^{2} m_{e} / \hbar^{2}$, respectively, we define the corresponding dimensionless quantities as

$\tilde{m}=\frac{m}{m_{e}}, \quad \tilde{r}=\frac{m_{e} \alpha}{\hbar^{2}} r, \quad \tilde{E}=\frac{\hbar^{2}}{m_{e} \alpha^{2}} E$,

$\tilde{n}_{e}=\left(\frac{\hbar^{2}}{m_{e} \alpha}\right)^{2} n_{e}$

Performing the integration in (1), we obtain

$w_{t}(\tilde{E}, l)=\exp \left[-\sqrt{8}\left(L_{2}-L_{1}\right)\right]$

$L_{1}=\tilde{r}_{0} \sqrt{\frac{l(l+1)}{2 \tilde{r}_{0}^{2}}+\frac{1}{\tilde{r}_{0}}-\tilde{E}}-$

$-\frac{1}{2 \tilde{E}} \arcsin \left(\frac{1-2 \tilde{E} \tilde{r}_{0}}{\sqrt{1+2 \tilde{E} l(l+1)}}\right)-\sqrt{\frac{l(l+1)}{2}} \times$

$\times \ln \left(\frac{1+l(l+1) / \tilde{r}_{0}+\sqrt{2 l(l+1)} \sqrt{\tilde{E}-l(l+1) / 2 \tilde{r}_{0}^{2}-1 / \tilde{r}_{0}}}{\sqrt{1+2 \tilde{E} l(l+1)}}\right)$,

$L_{2}=\frac{\pi}{4 \sqrt{\tilde{E}}}+\frac{i \pi}{2} \sqrt{\frac{l(l+1)}{2}}$.

[Note that $w_{t}$ is a real quantity, as the imaginary parts of $L_{1}$ and $L_{2}$ cancel each other.] A representative plot of $w_{t}(\tilde{E}, l)$ for a fixed $l>0$ is shown in Fig. 1 .

The balance of the total (classical and tunneling) electron current and the (classical) ion current $I^{(i)}=$ $-\sqrt{8 \pi} e r_{0}^{2} n_{i} v_{T i}\left(1-e \phi_{s} / T_{i}\right)$ [2] onto the grain defines the equilibrium surface potential $\phi_{s}=\alpha / r_{0}$ of the grain. The corresponding equation of balance of currents,

$I_{\text {clas }}^{(e)}+I_{\text {tun }}^{(e)}=-I^{(i)}$,

is a nonlinear equation for the surface potential of the grain (and hence for the grain charge $Z_{d}$, since $\phi_{s}=$ $\alpha / r_{0}$ with $\alpha=e^{2} Z_{d}$ ) that can be solved iteratively, for a given distribution function $f_{e}^{(-)}(E, l)$ of incoming plasma electrons far from the grain.

The importance of the electron quantum tunneling onto the grain is characterized by the ratio of the tunneling and classical electron currents $\eta_{I}=I_{\text {tun }}^{(e)} / I_{\text {clas }}^{(e)}$. The tunneling electron accretion current increases the total 
electron current onto the grain, thus offsetting the total electron-ion current balance and changing the equilibrium grain charge. This change is characterized by the ratio $\eta_{Z}=\left(Z_{d}^{\text {clas+tun }}-Z_{d}^{\text {clas }}\right) / Z_{d}^{\text {clas }}$ of the grain charges defined from (8) with and without accounting for the electron tunneling current. The ratios $\eta_{I}$ and $\eta_{Z}$ depend on several factors: the electron distribution function, electron and ion temperatures, the grain size, and the type of gas used in the plasma discharge. These dependences are illustrated in Tables 1 and 2, respectively, for the following three "model" electron distribution functions:

1. Maxwellian distribution:

$f_{e}^{(-)}(E, l)=f_{\mathrm{M}}(E)=n_{0 e}\left(\frac{m_{e}}{2 \pi T_{e}}\right)^{3 / 2} \exp \left(-\frac{E}{T_{e}}\right)$

where $n_{0 e}$ is the plasma electron density far from the grain, $T_{e}$ is the electron temperature in units of energy. 2. Druyvesteyn distribution:

$f_{e}^{(-)}(E, l)=f_{\mathrm{D}}(E)=$

$=n_{0 e} A_{\mathrm{D}}\left(\frac{m_{e}}{\pi T_{e}}\right)^{3 / 2} \exp \left[-B_{\mathrm{D}}\left(\frac{E}{T_{e}}\right)^{2}\right]$

where the constants $A_{\mathrm{D}} \approx 0.177$ and $B_{\mathrm{D}} \approx 0.243$ are defined from the conditions $\left\langle n_{e}\right\rangle=n_{0 e}$ and $\langle E\rangle=(3 / 2) T_{e}$ (here $\langle\ldots\rangle$ denotes the average over all electrons). This type of electron distribution typically takes place in gas discharges. For example, experimental measurements in inductively coupled plasma (ICP) [10] and capacitively coupled plasma (CCP) [11] discharges revealed that the electron distribution function at low pressures is indeed close to Maxwellian. At higher pressures ( $p \gtrsim 10$ mTorr in ICP and $p \gtrsim 0.5$ Torr in CPP), it turns into a Druyvesteyn-like distribution with a depleted high-energy tail.]

3. Step-like distribution: in complex plasmas, dust grains absorb electrons with energies above $U_{\text {eff }}\left(r_{0}, J^{2}\right)$, which depletes the high-energy "tail" of the electron distribution function [12]. For high enough number density of the grain component, this effect becomes significant, and the resulting electron distribution function can be roughly approximated by the step function

$f_{e}^{(-)}(E, l)=f_{\mathrm{S}}(E)=$

$\begin{cases}\frac{3 \sqrt{2} n_{0 e}}{16 \pi}\left(\frac{m_{e}}{E_{\max }}\right)^{3 / 2} & \text { for } E \leq E_{\max } \\ 0 & \text { for } E>E_{\max }\end{cases}$

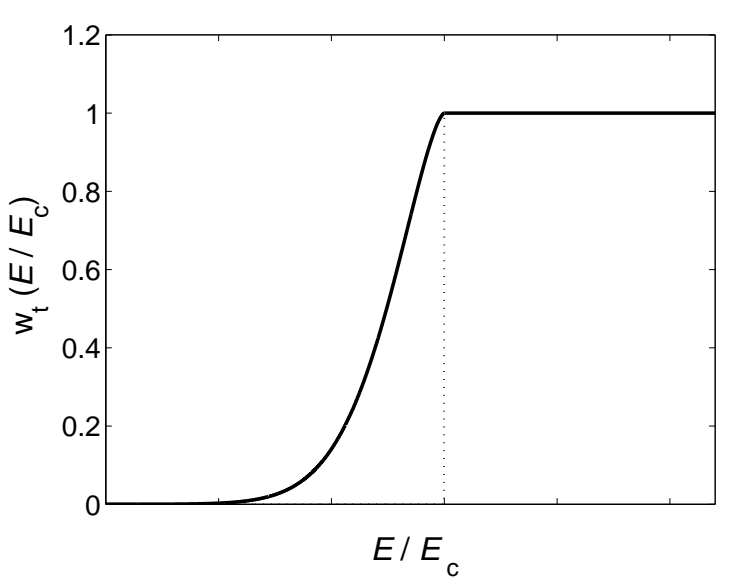

Dependence of tunneling probability $w_{t}$ on the normalized electron energy $\tilde{E}=E / E_{c}$ (where $E_{c}=\alpha^{2} m_{e} / \hbar^{2}$ is the energy in Coulomb units) for a fixed value of $l>0$

Here, $E_{\max }=e\left|\phi_{s}\right|+\delta E$, where $e\left|\phi_{s}\right|$ is the minimum energy, at which an electron with $l=0$ (zero angular momentum with respect to the grain) hits the grain, and $\delta E$ accounts for the fact that most electrons have $l>0$. For simplicity, $\delta E$ can be approximated by a constant defined, e.g., from experimental data for a particular complex plasma system. Here, for definiteness, we assume a reasonable value $\delta E=0.5 T_{e}$.

Generally, since $\eta_{I}$ is proportional to the ratio of the populations of electrons with $E \geq U_{\text {eff }}\left(r_{0}, l\right)$ and $E<U_{\text {eff }}\left(r_{0}, l\right)$, both $\eta_{I}$ and $\eta_{Z}$ increase for electron distributions with depleted tails, such as the Druyvesteyn or step distribution. Due to the same reason, for a given type of electron distribution function, $\eta_{I}$ and $\eta_{Z}$ increase at lower electron temperatures $T_{e}$ (for a fixed ion temperature $T_{i}$ ) and increase for smaller grain sizes $r_{0}$.

The criterion of validity of the quasiclassical approximation used here is reduced to the requirement that the electron's de Broglie wavelength $\lambda \sim \hbar / \sqrt{2 m_{e}|E|}$ is small compared to the size $\alpha /|E|$ of the region near the grain, where the electron energy $E$ is of the order of $U_{\text {eff }}$ [7]. For $l=0$ (when this criterion is the strongest, since $U_{\text {eff }}$ is the smallest for electrons with $l=0$ and increases with $l$ ), it becomes $\hbar v / \alpha \ll 1$, where $v \sim \sqrt{E / m_{e}}$ is the classical electron velocity. With $v \sim v_{T e}=\sqrt{T_{e} / m_{e}}$ and $\alpha \sim r_{0} z T_{e}$, where $z=e\left|\phi_{s}\right| / T_{e} \sim 1$ is the dimensionless grain charge, we have the following requirement for the applicability of the quasiclassical approximation for the electron motion in the grain field:

$r_{0} \gg \frac{\hbar}{\sqrt{m_{e} T_{e}}}$. 
T a b l e 1. Ratio $\eta_{I}=I_{\text {tun }}^{(e)} / I_{\text {clas }}^{(e)}$, for different electron distribution functions, electron temperatures, and grain sizes, in argon plasma with $T_{e} / T_{i}=10^{2}$

\begin{tabular}{|c|c|c|c|c|c|c|c|c|c|}
\hline \multirow{3}{*}{$\eta_{I}$} & \multicolumn{3}{|c|}{ Maxwellian } & \multicolumn{3}{|c|}{ Druyvesteyn } & \multicolumn{3}{|c|}{ Step distribution } \\
\hline & \multicolumn{3}{|c|}{ Grain radius $r_{0}, \mathrm{~cm}$} & \multicolumn{3}{|c|}{ Grain radius $r_{0}, \mathrm{~cm}$} & \multicolumn{3}{|c|}{ Grain radius $r_{0}, \mathrm{~cm}$} \\
\hline & $10^{-6}$ & $10^{-5}$ & $10^{-4}$ & $10^{-6}$ & $10^{-5}$ & $10^{-4}$ & $10^{-6}$ & $10^{-5}$ & $10^{-4}$ \\
\hline$T_{e}=0.01 \mathrm{eV}$ & 0.33 & 0.05 & 0.01 & 2.0 & 0.22 & 0.04 & 5.0 & 0.43 & 0.08 \\
\hline$T_{e}=0.1 \mathrm{eV}$ & 0.12 & 0.02 & 0.005 & 0.57 & 0.10 & 0.02 & 1.24 & 0.18 & 0.04 \\
\hline$T_{e}=1 \mathrm{eV}$ & 0.05 & 0.01 & 0.002 & 0.22 & 0.04 & 0.01 & 0.43 & 0.08 & 0.02 \\
\hline
\end{tabular}

T a b l e 2. Ratio $\eta_{Z}=\left(Z_{d}^{\text {clas+tun }}-Z_{d}^{\text {clas }}\right) / Z_{d}^{\text {clas }}$ for different electron distribution functions, electron temperatures, and grain sizes in argon plasma with $T_{e} / T_{i}=10^{2}$

\begin{tabular}{|c|c|c|c|c|c|c|c|c|c|}
\hline \multirow{3}{*}{$\eta_{Z}$} & \multicolumn{3}{|c|}{ Maxwellian } & \multicolumn{3}{|c|}{ Druyvesteyn } & \multicolumn{3}{|c|}{ Step distribution } \\
\hline & \multicolumn{3}{|c|}{ Grain radius $r_{0}, \mathrm{~cm}$} & \multicolumn{3}{|c|}{ Grain radius $r_{0}, \mathrm{~cm}$} & \multicolumn{3}{|c|}{ Grain radius $r_{0}, \mathrm{~cm}$} \\
\hline & $10^{-6}$ & $10^{-5}$ & $10^{-4}$ & $10^{-6}$ & $10^{-5}$ & $10^{-4}$ & $10^{-6}$ & $10^{-5}$ & $10^{-4}$ \\
\hline$T_{e}=0.01 \mathrm{eV}$ & 0.1 & 0.02 & 0.03 & 0.25 & 0.05 & 0.01 & 1.25 & 0.18 & 0.04 \\
\hline$T_{e}=0.1 \mathrm{eV}$ & 0.03 & 0.007 & 0.002 & 0.1 & 0.02 & 0.005 & 0.45 & 0.08 & 0.02 \\
\hline$T_{e}=1 \mathrm{eV}$ & 0.02 & 0.003 & $<0.001$ & 0.05 & 0.01 & 0.002 & 0.18 & 0.04 & 0.008 \\
\hline
\end{tabular}

This gives $r_{0} \gg 10^{-8} \mathrm{~cm}$ for $T_{e} \sim 1 \mathrm{eV}$ and $r_{0} \gg$ $3 \times 10^{-7} \mathrm{~cm}$ for $T_{e} \sim 0.01 \mathrm{eV}$. In other words, the criterion of applicability of the quasiclassical approximation is satisfied for all the grain sizes used in Tables 1 and 2 .

As is seen from Tables 1 and 2, the effect of electron quantum tunneling from plasma onto the grain on the grain charging is small under the typical conditions of most lab-based experiments on dusty plasmas (micronsized grains in plasma with electron temperatures of few $\mathrm{eV})$ and only becomes significant for small grains $\left(r_{0} \sim\right.$ tens to hundreds of $\mathrm{nm}$ ) in plasmas with low electron temperatures $\left(T_{e} \sim\right.$ tens to hundreds of $\left.\mathrm{K}\right)$, especially for electron distributions with depleted high-energy "tails". Note that we underestimated the tunneling current by assuming the grain field to be unscreened. In reality, the grain is shielded by plasma, and its potential decays faster than the Coulomb potential, narrowing the width of the potential barrier around the grain and thus further increasing the electron tunneling probability and the electron accretion current onto the grain, especially in low temperature plasmas with small Debye lengths. An estimate shows that the further enhancement of the electron tunneling current due to the screening of the grain becomes important when

$\frac{r_{0}}{\lambda_{\mathrm{D}}} \gtrsim 1$

where $\lambda_{\mathrm{D}}$ is the plasma Debye length, and $\lambda_{\mathrm{D}} \sim \lambda_{D i}=$ $\sqrt{T_{i} / 4 \pi e^{2} n_{0}}$. The ratio $\Delta I_{\text {tun }}^{\mathrm{D}} / I_{\text {tun }}^{\mathrm{C}}=\left(I_{\text {tun }}^{\mathrm{D}}-I_{\text {tun }}^{\mathrm{C}}\right) / I_{\text {tun }}^{\mathrm{C}}$ (where $I_{\text {tun }}^{\mathrm{D}}$ and $I_{\text {tun }}^{\mathrm{C}}$ are the tunneling electron accretion currents onto the Debye-screened and Coulombscreened (i.e., unscreened) grains, respectively), charac- terising the significance of the enhancing effect of Debye screening of the grain on the tunneling current, is shown in Table 3 for various ratios $r_{0} / \lambda_{\mathrm{D}}$. It is seen that, for the plasmas with low electron and ion temperatures, this screening-induced enhancement of the tunneling current is rather noticeable, especially for larger grains in plasma with the Druyvesteyn electron distribution, by further increasing the role of the tunneling effect in the grain charging. For higher temperatures and/or lower plasma densities, however, the enhancing effect of screening reduces as $\sqrt{T_{i} / n_{0 e}}$, according to (10) (for plasmas with $\left.T_{i} \ll T_{e}\right)$.

Therefore, we expect the considered effect of quantum tunneling of plasma electrons onto the grain to be important in plasmas with nano- and submicron-sized dust grains [1], in ultracold plasmas (where the electron

$\mathrm{T}$ a b l e 3. Ratio $\Delta I_{\text {tun }}^{\mathrm{D}} / I_{\text {tun }}^{\mathrm{C}}=\left(I_{\text {tun }}^{\mathrm{D}}-I_{\text {tun }}^{\mathrm{C}}\right) / I_{\text {tun }}^{\mathrm{C}}$ (where $I_{\text {tun }}^{\mathrm{D}}$ and $I_{\text {tun }}^{\mathrm{C}}$ are tunneling electron accretion currents onto the Debye-screened and Coulomb-screened (i.e., unscreened) grains, respectively) for different electron distribution functions, and grain sizes. The currents are calculated for grains with the corresponding equilibrium charges defined by Eq. (8). These charges are immersed in argon plasma with $n_{0 e}=10^{12} \mathrm{~cm}^{-3}$, $T_{e}=0.01 \mathrm{eV}, T_{e} / T_{i}=10^{2}$, with the Debye length $\lambda_{\mathrm{D}} \sim \lambda_{D i} \sim 7.4 \times 10^{-6} \mathrm{~cm}$

\begin{tabular}{c|ccc}
\hline \multirow{2}{*}{$\Delta I_{\text {tun }}^{\mathrm{D}} / I_{\text {tun }}^{\mathrm{C}}$} & \multicolumn{4}{|c}{ Grain radius $r_{0}$ (and the corresponding $\left.r_{0} / \lambda_{\mathrm{D}}\right)$} \\
\cline { 2 - 4 } & $\begin{array}{c}10^{-6} \mathrm{~cm} \\
\left(r_{0} / \lambda_{\mathrm{D}}=0.14\right)\end{array}$ & $\begin{array}{c}10^{-5} \mathrm{~cm} \\
\left(r_{0} / \lambda_{\mathrm{D}}=1.4\right)\end{array}$ & $\begin{array}{c}10^{-4} \mathrm{~cm} \\
\left(r_{0} / \lambda_{\mathrm{D}}=14\right)\end{array}$ \\
\hline Maxwellian & 0.09 & 0.53 & 3.5 \\
Druyvesteyn & 0.13 & 0.68 & 4.5 \\
\hline
\end{tabular}


temperature can be as low as $30 \mathrm{~K}[8]$ ), and in dark molecular clouds in astrophysics [6,9].

The authors thank A. Samarian for useful discussions. This work was supported by the Australian Research Council.

1. S.V. Vladimirov and K.N. Ostrikov, Phys. Rep. 393, 175 (2004).

2. V.E. Fortov, A.G. Khrapak, S.A. Khrapak, V.I. Molotkov, and O.F. Petrov, Physics-Uspekhi 47, 447 (2004).

3. P.K. Shukla and B. Eliasson, Rev. Mod. Phys. 81, 25 (2009).

4. J. E. Allen, Phys. Scripta 45, 497 (1992).

5. Y.P. Raizer, Gas Discharge Physics (Springer, Berlin, 1991).

6. D.R. Flower and M.J. Middleton, Mon. Not. R. Astron. Soc. 352, 837 (2004).

7. L.D. Landau and E.M. Lifshits, Quantum Mechanics (Pergamon, New York, 1980).

8. J.L. Roberts, C.D. Fertig, M.J. Lim, and S.L. Rolston, Phys. Rev. Lett. 92, 253003 (2004).

9. B.P. Pandey and S.V. Vladimirov, Astrophys. J. 664, 942 (2007).
10. V.A. Godyak and R.B. Piejak, Phys. Rev. Lett. 65, 996 (1990).

11. M.S. Barnes, J.C. Forster, and J. H. Keller, Appl. Phys. Lett. 62, 2622 (1993)

12. V.N. Tsytovich, G.E. Morfill, S.V. Vladimirov, and H. Thomas, Elementary Physics of Complex Plasmas (Springer, Berlin, 2008).

Received 19.01.11

ПРО РОЛЬ КВАНТОВОГО ТУНЕЛЮВАННЯ

ЕЛЕКТРОНА В ЗАРЯДЦІ ПИЛОВИХ

ЧАСТИНОК У КОМПЛЕКСНІЙ ПЛАЗМІ

Ю.О. Тищецъкий, С.В. Владимиров

Р е з ю м е

У квазікласичному наближенні проаналізовано вплив квантового тунелювання на струм акреції електронів плазми на від'ємно заряджену частинку, занурену в електронну плазму, для різних функцій розподілу електронів у плазмі, параметрів плазми і розмірів частинок. Показано, що внесок квантового тунелювання в зарядку частинок нехтовно малий для відносно великих (мікронних розмірів) пилових частинок у плазмі 3 температурою електронів порядку декількох еВ, але стає значним для наночастинок (діаметром в десятки-сотні нм) у холодних і ультрахолодних плазмах з температурою електронів десятки-сотні градусів Кельвіна, особливо у плазмах з малозаселеним високоенергетичним хвостом розподілу електронів за енергією. 